\title{
End-point energy measurements of field emission current in a continuous-wave normal-conducting rf injector
}

\author{
D. C. Nguyen,* N. A. Moody, H. L. Andrews, G. Bolme, L. J. Castellano, C. E. Heath, F. L. Krawczyk, \\ S. I. Kwon, R. McCrady, F. A. Martinez, P. Marroquin, M. Prokop, R. M. Renneke, P. Roybal, \\ W. T. Roybal, T. L. Tomei, P. A. Torrez, W. M. Tuzel, and T. Zaugg \\ Los Alamos National Laboratory, Los Alamos, New Mexico 87545, USA
}

(Received 20 September 2010; published 17 March 2011)

\begin{abstract}
The LANL/AES normal-conducting radio-frequency injector has been tested at $\mathrm{cw}$ cathode gradients up to $10 \mathrm{MV} / \mathrm{m}$. Field-emission electrons from a roughened copper cathode are accelerated to beam energy as high as $2.5 \mathrm{MeV}$ and impinge on a stainless steel target. The energies of the resulting bremsstrahlung photons are measured at varying levels of injector cavity rf power corresponding to different accelerating gradients. At low cavity power, the bremsstrahlung spectra exhibit well-defined endpoint energies at the positions where the number of single-photon events decreases to one $(\mathrm{S} / \mathrm{N}$ ratio $=1)$. Increasing the cavity power raises the probability of two-photon events in which two photons simultaneously arrive at the detector and register counts at twice the photon energy. The end-point energies at high cavity power are recorded at positions where the single-photon events transition to two-photon events. The measured end-point energies using this method are in excellent agreement with PARMELA calculations based on the cavity gradients deduced from the cavity rf power measurements.
\end{abstract}

DOI: 10.1103/PhysRevSTAB.14.030704

PACS numbers: 41.85.Ar, 41.60.Cr

\section{INTRODUCTION}

Recent success of the Linac Coherent Light Source testifies to the importance of high-brightness electron beams to the performance of $\mathrm{x}$-ray free-electron lasers (XFEL). In an XFEL, a high-gradient electron injector produces electron beams at bunch charge of $0.1-1 \mathrm{nC}$ and normalized rms emittance of $1 \mu \mathrm{m}$ or less, followed by acceleration to $\mathrm{GeV}$ beam energy and compression in magnetic chicane to produce fs electron bunches with peak current of a few $\mathrm{kA}$ [1]. High-brightness normalconducting radio-frequency (NCRF) injectors employ picosecond laser pulses to produce short electron bunches in phase with an rf accelerating field that quickly accelerates the electrons to relativistic energies. The combination of high accelerating gradients and magnetic solenoid emittance compensation [2] reduce emittance growth due to space charge and rf effects. Recent efforts in injector development have been focused on maximizing the cathode accelerating gradients while increasing the duty factor [3].

The high-gradient, high-brightness NCRF injectors are limited to low-duty-factor operation to keep rf power consumption and heating under control. Extending the duty factor of these high-brightness injectors introduces technical challenges in terms of ohmic losses, dark current, and

\footnotetext{
*dcnguyen@lanl.gov
}

Published by American Physical Society under the terms of the Creative Commons Attribution 3.0 License. Further distribution of this work must maintain attribution to the author(s) and the published article's title, journal citation, and DOI. the size and complexity of the photocathode drive lasers. Since ohmic losses scale with the square of accelerating gradients, heat removal from rf surfaces becomes exceedingly difficult for high-gradient, high-duty-factor operation. Another problem posed by high gradients is the field-emission (dark) current that scales nonlinearly with cathode gradient. To minimize dark current, high-gradient injectors use metal photocathodes that only respond to high-energy UV photons. The quantum efficiency of a typical metal photocathode is at least 3 orders of magnitude below that of semiconductor photocathodes, and thus the lasers driving metal photocathodes are large and complex.

High-duty operation requires new designs of NCRF injectors that can operate with semiconductor photocathodes at relatively low gradients to minimize ohmic heating on the rf surfaces, but still produce $\mathrm{nC}$ electron bunches with good emittance. The first high-duty-cycle NCRF gun was the Boeing/LANL injector at $433 \mathrm{MHz}$. This gun consisted of two independently driven reentrant cavities operated at a cathode gradient of $26 \mathrm{MV} / \mathrm{m}$ with $25 \%$ duty factor. It delivered an average beam current of $32 \mathrm{~mA}$ and normalized rms emittance of $5 \mu \mathrm{m}$ at $1 \mathrm{nC}$ [4]. The second high-duty-cycle NCRF injector design was the $1 \%$ duty L-band gun at the Photoinjector Test at Zeuthen (PITZ) facility. The PITZ gun was tested at accelerating gradients up to $60 \mathrm{MV} / \mathrm{m}$ and produced $\mathrm{nC}$ electron bunches with normalized rms emittance of $1.25 \mu \mathrm{m}$ [5]. At these high gradients, dark current from the $\mathrm{Cs}_{2}$ Te photocathode was substantial, illustrating one of the weaknesses of semiconductor photocathodes [6]. 
Another design of high-duty-factor NCRF injector is the LBNL quarter-wave (QW), low-frequency gun [7]. The QW design offers a number of benefits such as good vacuum conductance and low rf power consumption. At $20 \mathrm{MV} / \mathrm{m}$ accelerating gradient, the $\mathrm{QW}$ injector can produce electron beam energy up to $750 \mathrm{keV}$. The maximum electron bunch repetition rate of the QW injector is set by its low resonance frequency, e.g., $187 \mathrm{MHz}$ for the LBNL QW gun. We previously reported the design of a continuous-wave (cw) $700 \mathrm{MHz}$ NCRF injector using tapered ridge-loaded waveguides as the rf power couplers [8,9]. This NCRF injector was fabricated by Advanced Energy System (AES). The results of lowpower $\mathrm{rf}$ tests on the LANL/AES injector were reported elsewhere [10].

A more desirable, but less mature, injector design for $\mathrm{cw}$ operation is the superconducting rf gun. High gradients can be achieved at very low ohmic losses in an SRF injector. Also, cryopumping at liquid helium temperature improves the vacuum which can potentially lead to very long photocathode lifetime. The main problem with the SRF guns is the exclusion of an external magnetic field via Meissner effect after the cavities have become superconducting. This precludes the use of solenoid magnetic field for emittance compensation. New ideas for emittance compensation, e.g., rf focusing, higher-order magnetic modes, and external superconducting solenoid, have been proposed but thus far $\mathrm{nC}$ bunch charge in a single rf cycle has not been measured [11-13]. Another issue with SRF guns is the potential incompatibility between the multialkali cathodes and the niobium superconducting surfaces, i.e., contamination of the superconducting surfaces by the materials coming off the photocathodes. New ideas of advanced cathodes such as the diamond amplifier cathode have been proposed as a workaround for the contamination problems [14]. To date, the highest bunch charge achieved with SRF injectors is less than $70 \mathrm{pC}$ [15].

We report the first cw (100\% duty factor) operation of the NCRF injector at cathode gradients up to $10 \mathrm{MV} / \mathrm{m}$ and experimental results that validate the existence of such gradients in the LANL/AES injector with field emission from a roughened metal cathode. The key to highbrightness beam generation at low gradients is to employ radial focusing of electron beams near the photocathode to compensate for space-charge induced radial expansion that would otherwise result in rf-induced emittance growth. Radial focusing of the electron beams is achieved with a solenoid magnetic field near the cathode (the main solenoid field at the photocathode is zeroed with a second bucking coil to avoid introducing angular momentum) and also with rf azimuthal magnetic field at small injection phases. Emittance compensation using an external solenoid magnetic field was analyzed based on plasma oscillation of the beam envelopes of different axial slices [16]. These beam envelopes rotate in phase space about an invariant envelope corresponding to a matched condition for a given beam energy and gradient. We show the feasibility of producing a low-emittance beam with relatively low accelerating gradients by properly matching the electron beam radius to the invariant envelope.

\section{BEAM DYNAMICS IN AN NCRF INJECTOR}

\section{A. Overcoming image charge field}

The cw, $2 \frac{1}{2}$-cell NCRF injector uses a solenoid magnetic field for radial focusing and emittance compensation, in lieu of ultrahigh accelerating gradients at the cathode, to produce low-emittance electron beams. Low emittance can be obtained by reducing the photoemission radius and increasing the solenoid magnetic field to keep the beam radius small throughout the injector. The emitted electrons create an electric field at the cathode and this resulting image charge field reduces the net accelerating field seen by subsequent electrons. The applied rf field at the time of photoemission needs to be sufficiently high to overcome the image charge. Otherwise, the electron bunch will exhibit temporal distortions characteristic of space-charge limited emission [17]. For a given injection phase, bunch charge, and photoemission radius, the following inequality must be satisfied:

$$
E_{0} \sin \phi_{\text {inj }}>\frac{Q}{\varepsilon_{0} \pi R^{2}},
$$

where $E_{0}$ is the peak gradient at the cathode, $\phi_{\text {inj }}$ the injection phase, $Q$ electron bunch charge, and $R$ photoemission radius. For instance, with $1 \mathrm{nC}$ bunch charge and $4 \mathrm{~mm}$ photoemission radius, the image charge field is $2.25 \mathrm{MV} / \mathrm{m}$. At $30^{\circ}$ injection phase and to satisfy the above inequality, the peak gradient at the cathode has to be at least $4.5 \mathrm{MV} / \mathrm{m}$. It is worth noting that this minimum gradient is much lower than the cathode fields typically found in pulsed, high-gradient NCRF injectors.

A diagram of the LANL/AES injector is shown in Fig. 1. The injector consists of 2.5 resonant cells and a nonreso-

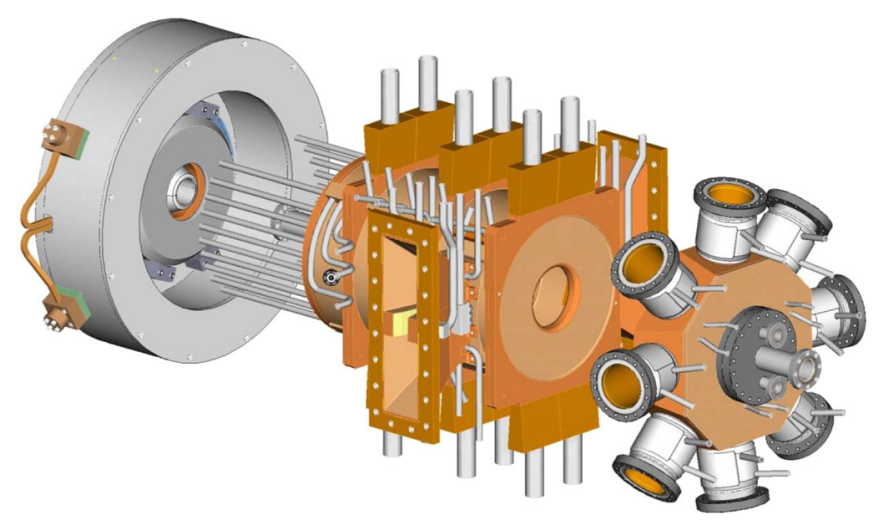

FIG. 1. Diagram of the LANL/AES normal-conducting rf injector. 
nant cell for vacuum pumping. The rf power is coupled into the cavity via two ridge-loaded waveguides. Two magnetic solenoids are mounted on the cathode cell to provide an external magnetic field for radial focusing and emittance compensation.

\section{B. Emittance compensation at low gradients}

The envelope equation of an axisymmetric electron beam inside an $\mathrm{rf}$ injector with slowly varying gradients can be written as follows [18]:

$$
\begin{aligned}
\sigma^{\prime \prime} & +\sigma^{\prime} \frac{\gamma^{\prime}}{\beta^{2} \gamma}+k_{B}^{2} \sigma-\frac{I}{2 \beta^{3} \gamma^{3} I_{0} \sigma}-\frac{e E_{r}}{\beta \gamma m c^{2}} \\
& +\frac{e v_{z} B_{\theta}}{\beta \gamma m c^{2}}-\frac{\varepsilon_{T}^{2}}{\beta^{2} \gamma^{2} \sigma^{3}}=0 .
\end{aligned}
$$

The first term denotes the second derivative of rms radius with respect to $z$; the second term denotes damping of the radial expansion due to acceleration ( $\beta$ is the electron velocity relative to the speed of light, $\gamma$ is the Lorentz factor, and prime denotes the first derivative with respect to $z$ ); the third term denotes radial focusing with an external magnetic field $\left(k_{B}=\frac{e B}{2 m c \beta \gamma}\right.$, where $e$ is the electron charge, $B$ the external magnetic field, $m$ the electron mass, and $c$ the speed of light). The fourth term denotes spacecharge induced radial expansion. Here, $I$ is the peak current and $I_{0}$ is $17 \mathrm{kA}$. Space charge causes the beam to expand into regions near the cavity apertures where it experiences the radial component of the rf field (fifth term). This timedependent rf kick causes rf-induced emittance growth [19].

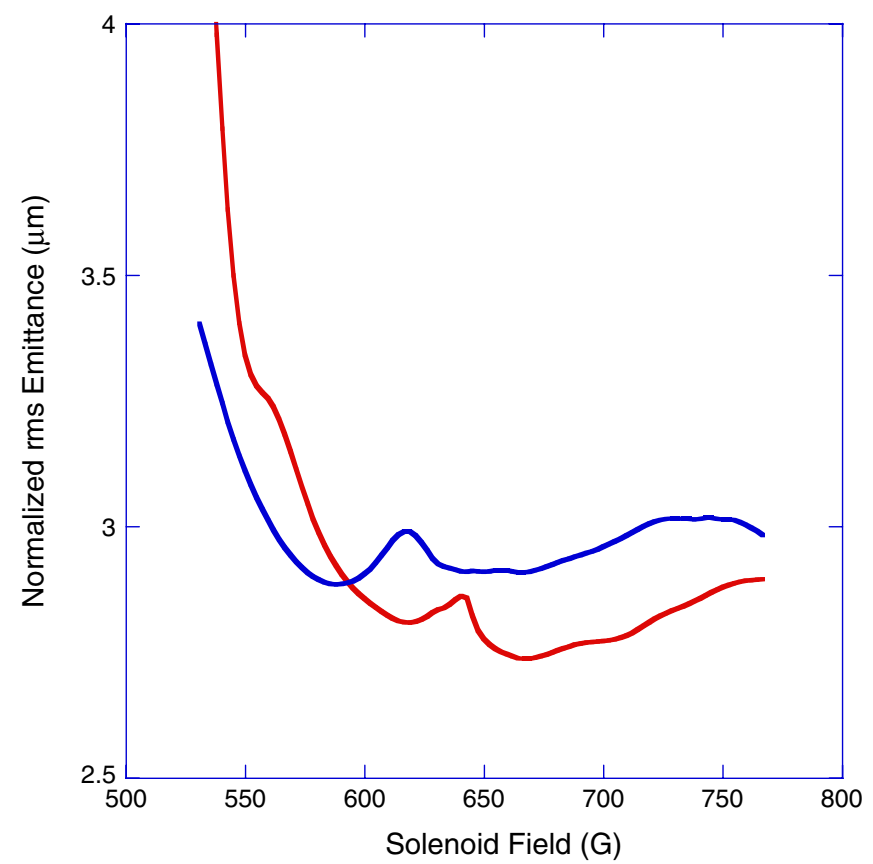

FIG. 2. Plots of calculated normalized rms emittance versus solenoid field at $9 \mathrm{MV} / \mathrm{m}$ (blue) and $10 \mathrm{MV} / \mathrm{m}$ (red) cathode gradients.
The sixth term corresponds to rf focusing at the cathode due to $\mathrm{v} \times \mathrm{B}$ force and provides additional focusing at small injection phases. The last term contains $\varepsilon_{T}$ which is the beam's thermal emittance due to residual transverse kinetic energy of the emitted electrons. With a combination of solenoid focusing [third term of Eq. (2)] and rf magnetic focusing (sixth term) at small launch phase, we can counteract the space-charge and rf-induced expansion (fourth and fifth terms) and minimize the need for damping with high accelerating gradients (second term).

Beam dynamics have been simulated using the phase and radial motion in electron linear accelerators (PARMELA) code. The normalized rms emittance for $1 \mathrm{nC}$ at two cathode gradients $(9$ and $10 \mathrm{MV} / \mathrm{m}$ ) is plotted as a function of the solenoid magnetic field in Fig. 2, with electron bunch length of 14 picoseconds (full width at half maximum). The minimum rms emittance for $1 \mathrm{nC}$ at $10 \mathrm{MV} / \mathrm{m}$ cathode gradient is $2.75 \mu \mathrm{m}$. This rms emittance can be reduced further by using a smaller photoemission radius and higher solenoid magnetic field at the expense of a longer electron bunch length, thus requiring higher compression ratio from the chicane bunch compressor.

\section{Field-emission dynamics}

Beam dynamics were simulated with PARMELA to predict the maximum energy of the field-emitted electrons exiting the injector at different cavity power levels. The maximum electron energy was observed at a launch phase of $40^{\circ}$ (the cathode field peaks at $90^{\circ}$ ). Electrons born at $40^{\circ}$ spend more time in the first cell and arrive at the centers of the second and third cells at the peak of the rf fields. Consequently, these electrons experience the highest acceleration throughout the 2.5-cell injector. At a cathode gradient of $9.8 \mathrm{MV} / \mathrm{m}$, the LANL/AES NCRF injector is capable of producing beam energy up to $2.5 \mathrm{MeV}$. With photoemission at an injection phase of $17^{\circ}$, corresponding to a cathode field of only $2.9 \mathrm{MV} / \mathrm{m}$, and a solenoid magnetic field of $660 \mathrm{G}$, PARMELA predicts a normalized rms emittance at $1 \mathrm{nC}$ of $2.75 \mu \mathrm{m}$. The goal of our experiment is to confirm this cathode gradient can be achieved continuously in the LANL/AES NCRF injector by independently measuring the end-point gamma energy from the electrons that are field emitted at the cathode and traverse the entire cavity.

Both field emission and secondary emissions in a highgradient rf gun have been studied recently [20]. Field emission follows the well-known Fowler-Nordheim equation, as shown below:

$$
J=A \frac{(\beta E)^{2.5}}{\Phi^{1.75}} \exp \left(\frac{-B \Phi^{3 / 2}}{\beta E}\right)
$$

where $J$ is the dark current density in $\mathrm{A} / \mathrm{m}^{2}, A$ is $1.5 \times 10^{6} \mathrm{eV}-\mathrm{A} / \mathrm{MV}^{2}, \quad \beta$ is the local electric field 
enhancement factor, $\phi$ is copper work function $(4.7 \mathrm{eV})$, and $B$ is $6830 \mathrm{MV} /\left(\mathrm{m}-\mathrm{eV}^{3 / 2}\right)$.

A combination of sinusoidal electric field and nonlinear dependence of field emission current create electron pulses with full width at half maximum shorter than one-half of an rf cycle (Fig. 3). The electron beam energy increases with launch phase up to $40^{\circ}$ and then drops off rapidly at about $60^{\circ}$ launch phase (Fig. 4). The PARMELA-generated energy

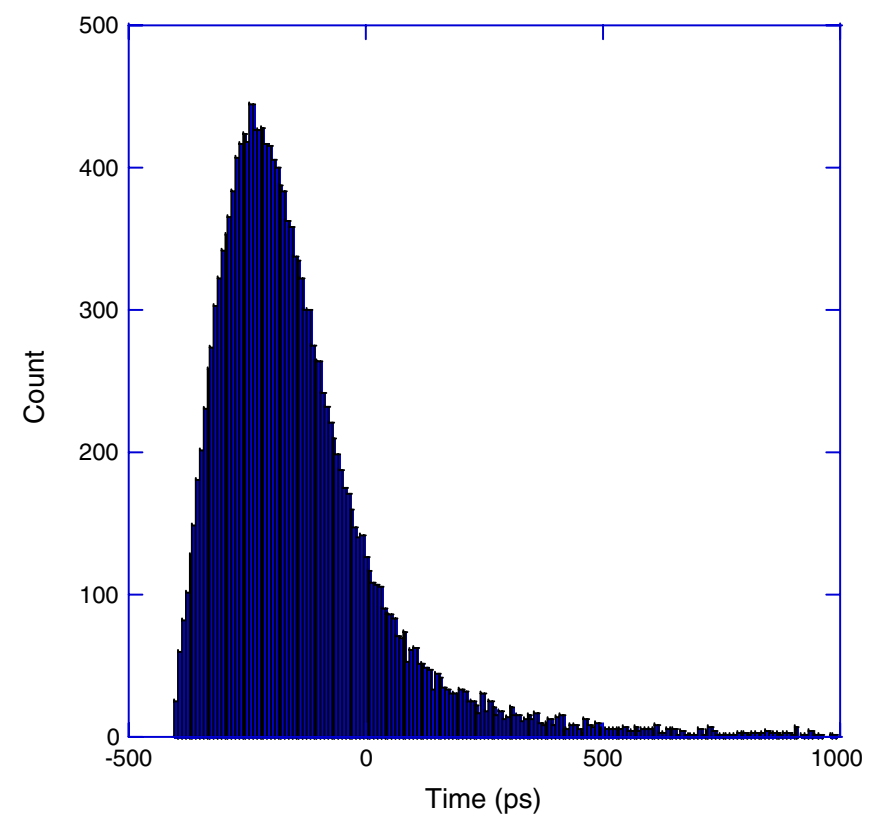

FIG. 3. PARMELA-predicted temporal profile of the fieldemission current pulse.

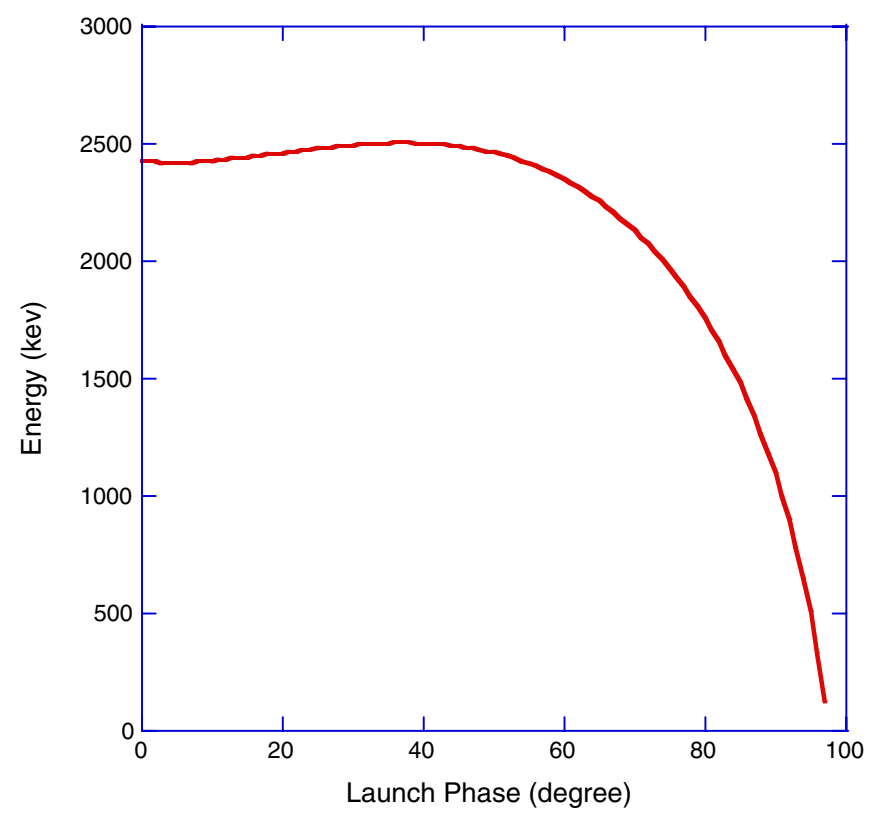

FIG. 4. Dependence of electron energy on $\mathrm{rf}$ phase of the injector cavity.

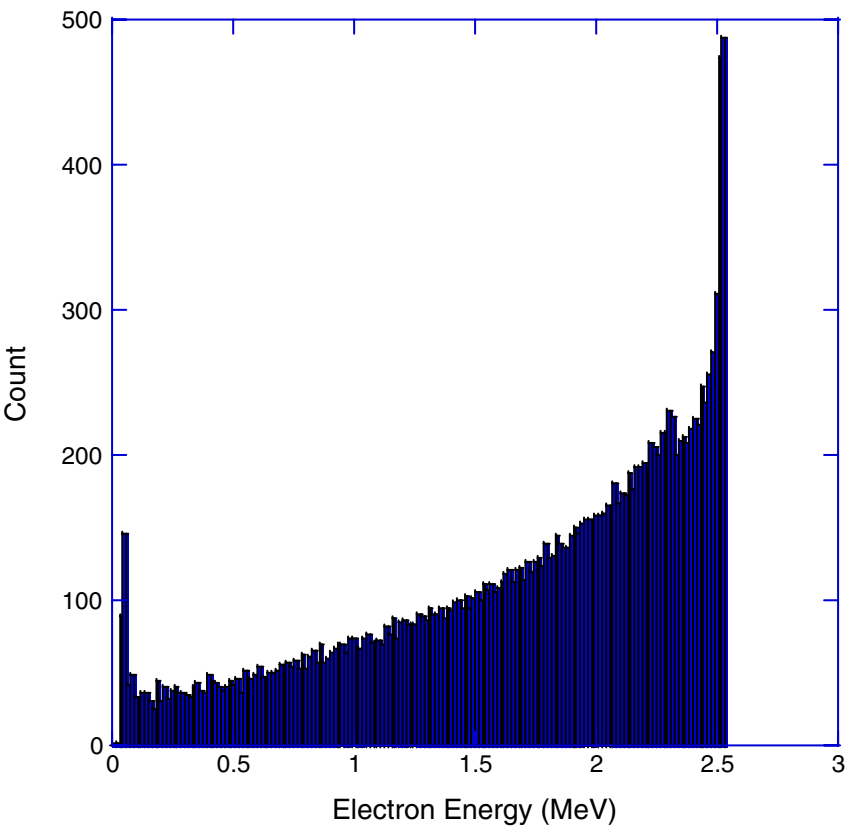

FIG. 5. PARMELA-predicted energy spectrum of field-emitted electrons at cavity pickup loop power of $702 \mathrm{~mW}$.

distribution of field-emitted electrons is plotted in Fig. 5, showing a well-defined maximum energy. This maximum energy scales linearly with cavity gradient (square root of cavity power). For this NCRF injector, the maximum energy can be approximated in terms of cavity pickup loop power as follows:

$$
E_{\max }=(3.0 \mathrm{MeV})\left(\frac{P_{\text {cavity }}}{1 \mathrm{~W}}\right)^{1 / 2} .
$$

\section{EXPERIMENT}

The NCRF injector cavity was powered by a previously reported rf system [21]. The cavity power was measured with rf pickup loops having $-60 \mathrm{~dB}$ coupling. The rf conditioning was performed with a rough copper cathode having machining grooves. These machining grooves provided the field-enhanced tips capable of field emission. The field-emitted electrons traversed the injector cavity axis and impinged on a stainless steel vacuum flange. Subsequent current measurement with a Bergoz wall current monitor recorded average dark current as high as $70 \mu \mathrm{A}$.

The bremsstrahlung photons emitted by the accelerated electrons impinging on the stainless steel flange were measured several meters downstream of the photoinjector by a Canberra high-purity germanium photon detector with relative efficiency of 5\%-8\%. The signals for the detected photons were amplified and shaped by a Tennelec spectroscopy amplifier for pulse height analysis. The pulse height was converted and stored by an EG\&G Ortec Model 918A analog-to-digital converter module and interfaced to a PC for viewing and data retrieval. 
Energy calibration for the converted photon spectrum was done with linear extrapolation between the $661.6 \mathrm{keV}$ peak of a ${ }^{137} \mathrm{Cs}$ source and the $1332.5 \mathrm{keV}$ peak of a ${ }^{60} \mathrm{Co}$ source. The combined detector system energy resolution for the end-point energy of the photoinjector data is on the order of $0.25 \%$. This does not represent the actual errors in reading the end-point energy which are affected by other experimental factors such as photon count rates and $\mathrm{rf}$ power fluctuations.

The gamma photon detector was positioned on the photoinjector axis approximately nine meters along the direction of the emitted electrons. A $\frac{1 / 1}{2}$ steel plate with an on-axis aperture was positioned near the photoinjector to attenuate the bremsstrahlung photons from peripheral regions of the accelerator cavities. The photon detector and liquid nitrogen cryostat were encased in a $2^{\prime \prime}$ lead enclosure with a $\frac{1}{4}$ ' diameter collimator to selectively pass only the on-axis photons. Because of the high rates of photon emission when the photoinjector was subject to high power rf, additional lead collimators with varying apertures down to $0.040^{\prime \prime}$ in diameter and up to $4 \frac{11 \prime}{4}$ thickness and lead absorbers up to $\frac{1}{8}$ in thickness were utilized to attenuate the photon count rates to levels manageable by the detector.

With the collimators and absorbers, the photon count rates were kept below the maximum count rate of $6000 \mathrm{~s}^{-1}$ at cavity pickup power up to $600 \mathrm{~mW}$. Above $600 \mathrm{~mW}$ pickup loop power, the count rate exceeded $12000 \mathrm{~s}^{-1}$ but reasonable end-point energies could still be extrapolated in spite of double-photon events at the high-energy end of the photon spectrum. The time interval was chosen such that the number of counts provided good statistics to establish an accurate reading of the end-point energy.

\section{RESULTS}

The end-point energies at low rf power are readily detectable from the photon energy where the photon count, $N_{\mathrm{ph}}$, is reduced to one. The error in the number of photon count is $\sqrt{N_{\text {ph }}}$ so the signal-to-noise ratio is also 1 when $N_{\mathrm{ph}}=1$. This corresponds to the limit of detection in photon counting. By reading the end-point energies at $N_{\mathrm{ph}}=1$ and taking the average, we can obtain the endpoint energy for a given power level. As can be seen in Fig. 6, the data points at $N_{\mathrm{ph}}=1$ have a mean value of $1449 \mathrm{keV}$ and standard deviation of $69 \mathrm{keV}$. The PARMELA predicted end-point energy is $1416 \mathrm{keV}$.

As rf power is increased, the photon count incident on the detector and the probability of photon pileup also increase. At high cavity rf power, the end-point energies must be taken, not at the point where $N_{\text {ph }}$ is equal to one but at the transitions from single-photon events to doublephoton events (Fig. 7). The measured counts at energies higher than $2.5 \mathrm{MeV}$ exhibit a different slope (less negative) than the single-photon events. The end-point energies using this method are plotted, together with PARMELA

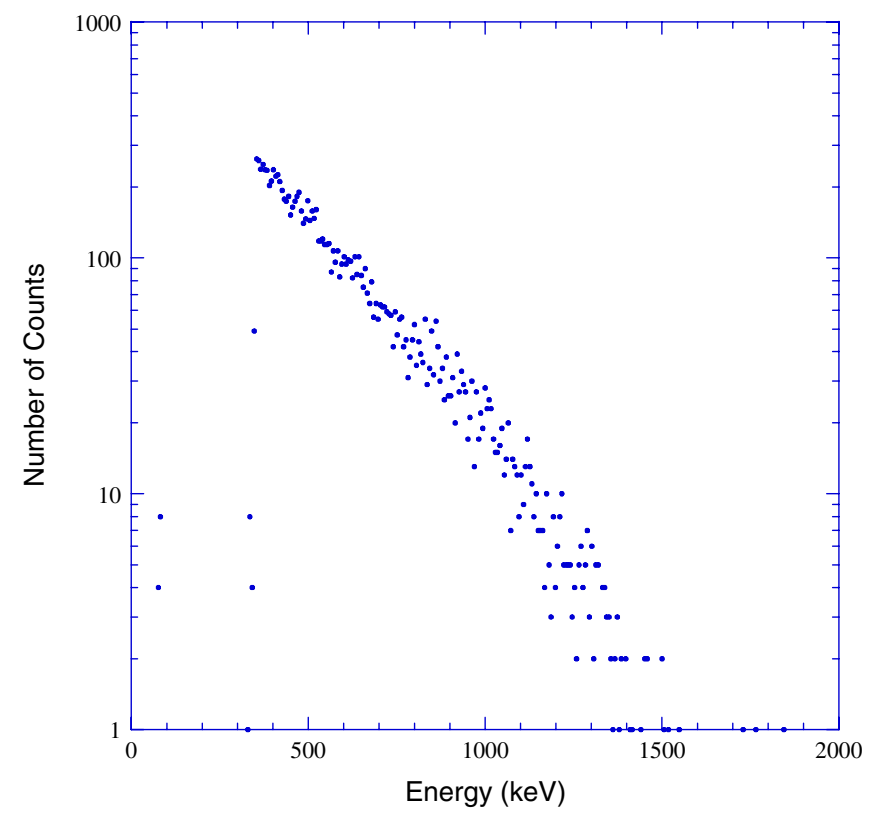

FIG. 6. Number of photons versus photon energy for experiment runs at $250 \mathrm{~mW}$ rf power. The mean energy of all data points at $N_{\text {ph }}=1$ is $1449 \mathrm{keV}$.

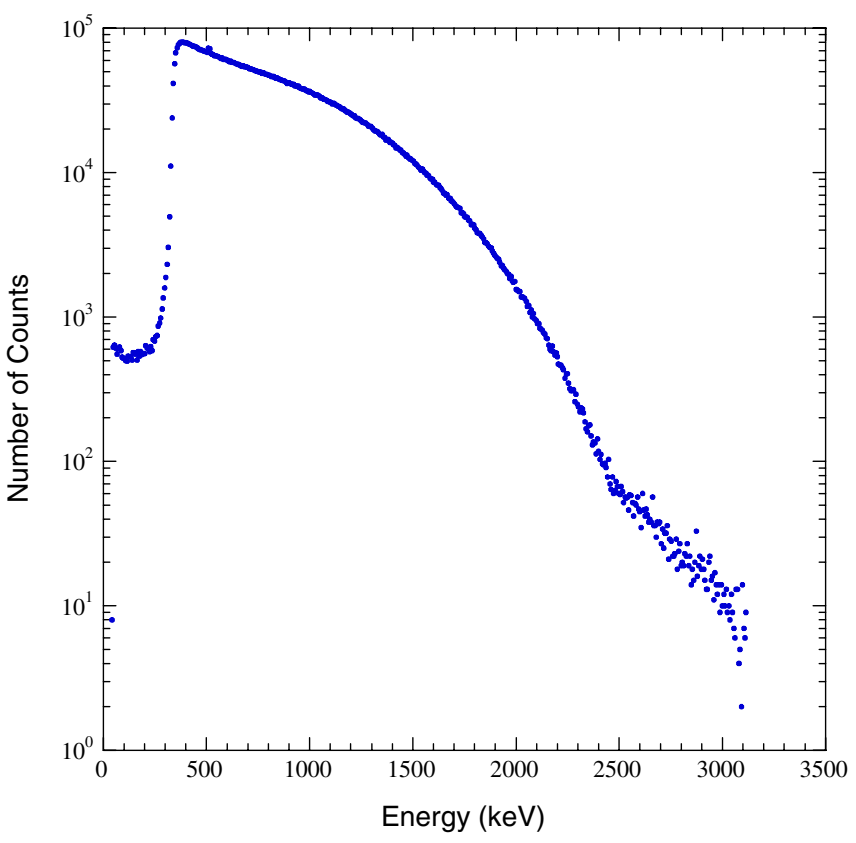

FIG. 7. Plot of photon counts versus energy at the highest power level showing the transition from single-photon events to two-photon events at $2.5 \mathrm{MeV}$.

predicted end points, versus cavity power in Fig. 8. The measured and PARMELA-predicted end-point energies agree over the entire range of $\mathrm{rf}$ power. Also plotted is a curve of end-point energies analytically calculated using Eq. (4). The analytic predictions agree with all experimental data except the lowest power level. The discrepancy is due to an 


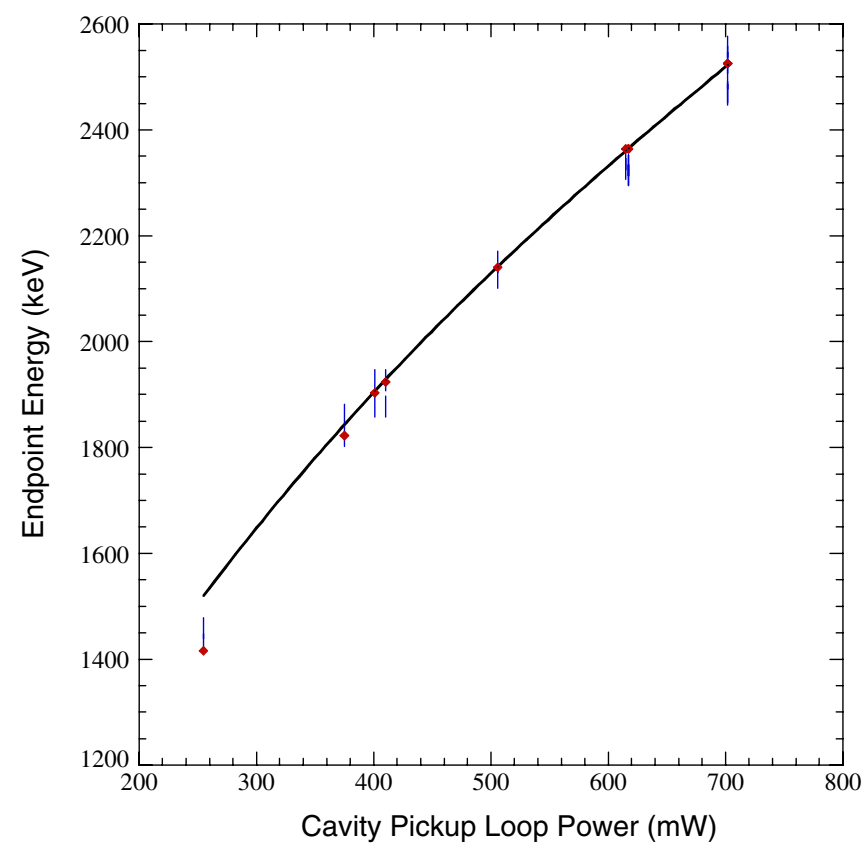

FIG. 8. End-point photon energy versus cavity power. The vertical bars are measured data \pm 2 standard deviations, the red circles are PARMELA prediction, and the line is analytic calculation.

incorrect assumption in the analytic model that the electrons are relativistic at this low accelerating gradient.

\section{CONCLUSIONS}

We report the first $\mathrm{cw}$ operation of a normal-conducting rf injector at cathode gradients up to $9.8 \pm 0.2 \mathrm{MV} / \mathrm{m}$. These gradients are adequate for accelerating $\mathrm{nC}$ bunches to beam energies of $2.5 \mathrm{MeV}$ without significant emittance dilution. With solenoid magnet emittance compensation, we expect a normalized rms emittance of $2.75 \mu \mathrm{m}$ for $1 \mathrm{nC}$.

Field emission is simulated with PARMELA at different cavity power levels to predict the maximum electron energies at each power level. As expected, the field-emission electrons exhibit broad energy spectra terminated with sharp cutoffs at the maximum energies.

End-point energy measurements have been performed at different rf power levels, corresponding to different cathode gradients. At low rf power, the heavily attenuated bremsstrahlung spectra exhibit sharp transitions corresponding to the maximum electron end-point energies. At higher cavity power, double-photon events increase the background counts at the high-energy end of the spectrum, and the maximum electron energies are recorded at the transitions from single-photon events to double-photon events. The measured end-point energies are in excellent agreement with PARMELA predictions using the accelerating gradients deduced from the measured cavity pickup loop power.

\section{ACKNOWLEDGMENTS}

We appreciate the financial support from the Office of Naval Research and the High Energy Laser Joint Technology Office. We also thank Lloyd Young for modifying the PARMELA code to model field emission in the NCRF injector.

[1] P. Emma, in Proceedings of the 23rd Particle Accelerator Conference, Vancouver, Canada, 2009 (IEEE, Piscataway, NJ, 2009).

[2] B. E. Carlsten, Nucl. Instrum. Methods Phys. Res., Sect. A 285313 (1989).

[3] R. Akre et al., Phys. Rev. ST Accel. Beams 11, 030703 (2008).

[4] D.H. Dowell et al., Appl. Phys. Lett. 63, 2035 (1993).

[5] F. Stephan et al., Phys. Rev. ST Accel. Beams 13, 020704 (2010).

[6] S. Lederer et al., in Proceedings of the 2007 Particle Accelerator Conference, Albuquerque, New Mexico (IEEE, Albuquerque, New Mexico, 2007).

[7] K. Baptiste et al., Nucl. Instrum. Methods Phys. Res., Sect. A 599, 9 (2009).

[8] D. C. Nguyen et al., Nucl. Instrum. Methods Phys. Res., Sect. A 528, 71 (2004).

[9] S. Kurrenoy et al., Nucl. Instrum. Methods Phys. Res., Sect. A 528, 392 (2004).

[10] F. Krawczyk et al., in LINAC08, XXIV Linear Accelerator Conference, Victoria, Canada, TUP18, http://accelconf.web.cern.ch/accelconf/LINAC08/papers/ tup108.pdf.

[11] D. Janssen et al., Nucl. Instrum. Methods Phys. Res., Sect. A 507, 314 (2003).

[12] K. Flottman, D. Janssen, and V. Volkov, Phys. Rev. ST Accel. Beams 7, 090702 (2004).

[13] R. Xiang et al., Phys. Rev. ST Accel. Beams 13, 043501 (2010).

[14] X. Chang et al., Phys. Rev. Lett. 105, 164801 (2010).

[15] F. Staufenbiel et al., Nucl. Instrum. Methods Phys. Res., Sect. A 584, 259 (2008).

[16] L. Serafini and J. B. Rosenzweig, Phys. Rev. E 55, 7565 (1997).

[17] S. M. Gierman, in Proceedings of the 2nd ICFA Advanced Accelerator Workshop on "The Physics of High-Brightness Electron Beams" (World Scientific, Singapore, 2000), pp. 511-526.

[18] M. Reiser, Theory and Design of Charged Particle Beam (Wiley-VCH Verlag GmbH \& Co. KGaA, Weinheim, 2008), 2nd ed.

[19] K-J. Kim, Nucl. Instrum. Methods Phys. Res., Sect. A 275, 201 (1989).

[20] J.H. Han et al., Phys. Rev. ST Accel. Beams 8, 033501 (2005).

[21] W. T. Roybal et al., in Proceedings of the 21st Particle Accelerator Conference, Knoxville, 2005 (IEEE, Piscataway, NJ, 2005), pp. 2413-2415. 